\title{
THE TREATMENT OF MALARIA
}

\author{
Sir Neil Hamilton Fairley, K.B.E., D.Sc., M.D., F.R.C.P., F.R.S. \\ Consulting Physician, The Hospital for Tropical Diseases, London, N.W. I
}

\section{Hyperinfection in Malignant Tertian Infection}

Where there is hyperinfection associated with cerebral features, where algid malaria is found, where the patient cannot swallow or when vomiting and diarrhoea are severe, anti-malaria drugs should be given intravenously. For this purpose, quinine hydrochloride gr. 10 in 10 to $20 \mathrm{ml}$. of isotonic saline may be slowly injected at the rate of I gr. per minute and repeated in six hours if necessary. The injection must be given slowly owing to the severe drop of blood pressure resulting from rapid injection. Never more than three injections should be given in the first 24 hours. If the blood pressure is unduly low or algid symptoms are present, quinine hydrochloride may be given slowly in one pint of saline rather than in concentrated form.

Chloroquine hydrochloride in a dosage of 200 to $300 \mathrm{mg}$. may be injected intramuscularly and repeated in eight hours, or $400 \mathrm{mg}$. of base in $500 \mathrm{ml}$. normal saline may be given slowly by intravenous drip, taking one hour for the injection. The sulphate in a dosage of $200 \mathrm{mg}$. base may be given intravenously and repeated in eight hours. Parenteral medication is stopped when the patient regains consciousness and when shock and symptoms of collapse improve.

It is often necessary in this condition to combat dehydration and to maintain blood volume by intravenous injections of isotonic saline ( 0.85 per cent.) or glucose ( 5 per cent.) given by continuous drip or intermittently.

Mepacrine hydrochloride (o.3 g.) may also be administered intramuscularly if other drugs given by the intravenous route are not available. A total of $1.0 \mathrm{~g}$. mepacrine parenterally should not be exceeded in the first 24 hours of treatment. Lumbar puncture with the withdrawal of $20 \mathrm{ml}$. of cerebrospinal fluid may be helpful. In addition, should hyperpyrexia be present, the naked patient is placed under a fan on a wire mattress and covered with a sheet sprayed with ice-cold water.
When the rectal temperature reaches $102^{\circ} \mathrm{F}$. hydrotherapy should be stopped.

Finally, if haemolytic anaemia be severe the patient should be transfused; in severe cases several pints of blood may be given. Oral medication with iron should follow.

\section{Ordinary M.T. Malarial Attack}

Bed rest during the febrile period and for at least three days after the temperature is normal is essential, especially in the first attack of malignant tertian malaria. Should there be anaemia, a pill containing ferrous sulphate (gr. 3 ) should be given thrice daily and if the anaemia proves severe, blood transfusion will help convalescence.

For M.T. malaria one of the more active schizonticidal drugs should be given orally. Proguanil (paludrine) and pyrimethamine (daraprim) though effective schizonticides, act too slowly in reducing the temperature and for this reason drugs like chloroquine sulphate or diphosphate and mepacrine hydrochloride are preferable.

Chloroquine sulphate or diphosphate is given orally to non-immunes in the following dosage: six tablets (or $900 \mathrm{mg}$.) on the first day; two tablets (or $300 \mathrm{mg}$.) on the second and third days. In partial immunes, four tablets (or $600 \mathrm{mg}$.) are given on one day only. Chloroquine is probably the best drug to use for the routine treatment of malaria.

Mepacrine hydrochloride is a very effective schizonticidal drug. It is given to non-immunes in a dosage of $300 \mathrm{mg}$. thrice daily for the first day, twice daily on the second day and then $100 \mathrm{mg}$. thrice daily for five days. Some 300 to $500 \mathrm{mg}$. of mepacrine are given to partial immunes in a single dose.

Quinine dihydrochloride or sulphate in a dosage of $\mathrm{r} O \mathrm{gr}$. thrice daily is given for five to seven days to non-immunes. This drug is not used so much now in chronic M.T. malaria owing to the danger of its precipitating blackwater fever. 
Lapudrine was discovered by Curd, Davey et al. in $195^{\circ} .^{2}$ It has one more chlorine atom than proguanil in the benzene ring and is more active than proguanil. It is more effective therapeutically and prevents the development of oocysts in the mosquito stomach.

\section{Radical Cure of Vivax and Quartan Malaria}

There is no evidence that in falciparum malaria late exo-erythrocytic forms persist. Once parasites have all been destroyed in the peripheral blood by schizonticidal drugs radical cure has been achieved; the so-called relapses are due either to the persistence of erythrocytic forms in sub-microscopic numbers in the peripheral blood or to reinfection. In vivax and quartan malaria, and possibly in $P$. ovale infections also, this is not the case. With benign tertian and quartan parasites late exoerythocytic forms often persist and produce relapses despite the previous total eradication of parasites in the circulating blood by schizonticidal drugs. We are dependent on the 8-aminoquinoline drugs for the radical cure of both these species of parasite. Pamaquine has been successfully used for this purpose for many years and more recently American workers have successfully produced several new 8-amino-quinoline drugs including pentaquine, iso-pentaquine and primaquine which are even more effective.

It was found that primaquine in a dosage of I 5 to $30 \mathrm{mg}$. daily for 14 days completely eradicated $P$. vivax parasites by destroying the persistent exo-erythrocytic forms in the liver. Toxic side-effects include gastro-intestinal disturbances, and haemolytic anaemia to which negroes are prone; the latter may be associated with haemoglobinuria. Concurrent administration of sulphadiazine and mepacrine may increase the toxicity of the 8-amino-quinolines, and for this reason must be avoided.

Reports were published on primaquine by authority of the Council on Pharmacy and Chemistry, U.S.A. ${ }^{11}$ If fever be present primaquine is given in combination with one of the schizonticidal drugs such as chloroquine or quinine but not mepacrine. Four groups of patients were treated. The first comprised 232 men who received chloroquine $\mathrm{I} .5 \mathrm{~g}$. in divided doses over three days; 64 patients ( 27 per cent.) relapsed. The second group of 246 men received the same dose of chloroquine plus $27 \mathrm{mg}$. primaquine daily as three divided doses for 14 days; one patient relapsed. The third group of 231 men received the same dosage of chloroquine and primaquine in a single daily dose of $15 \mathrm{mg}$. for I4 days; none relapsed and there were no significant toxic effects.

\section{Prevention of Transmission}

The 8-amino-quinolines in non-toxic dosage also destroy the sexual forms of $P$. falciparum ind the peripheral blood and the administration of ${ }_{C}$ pamaquine $10 \mathrm{mg}$. t.d.s. or of primaquine $7 \mathrm{mg}$. t.d.s. for two or three days are effective from thises viewpoint. Proguanil, lapudrine and pyrimetha-o mine do not destroy the gametocytes in the bloodo stream, but they do prevent their development in the mosquitoes' stomach so that infection of the $\underset{\complement}{\mathbb{\Phi}}$ salivary glands does not occur. In consequence, mosquitoes feeding on patients taking these drugs in appropriate dosage do not transmit the disease to others.

\section{Causal Prophylaxis and Suppression}

In experimentally infected volunteers in Cairns it was found with the South West Pacific strains byco Fairley et al..$^{\tilde{5}}$ that quinine (gr. Io) daily failed toi suppress malignant tertian malaria and a propor -0 tion of benign tertian infections also. Sulphadia- $-\dot{\omega}$ zine proved reasonably satisfactory in a dosage of r.o g. daily in M.T. infections, but overt attacks of B.T. malaria very frequently occurred.

When mepacrine (o.I g.) was given every day invariably, it was found that malignant tertian in-0 fections were both suppressed and radically cured@ and that benign tertian infections were suppressgd $\overrightarrow{-}$ throughout the period of mepacrine administ tion. Benign tertian relapses, however, generalsy occurred from two to seven weeks after the drụgo was stopped, but malignant tertian malaria nevers reappeared.

Factors generally regarded as precipitatingڤ relapses in latent malaria were also investigated. These included exposure to wet and cold, physical $\overrightarrow{0}$ exhaustion, anoxia and chill as in high altitude 3 flying, blood loss, anaesthetics, surgical operations and alcoholic excesses and strong emotions of anger and fear associated with outpouring ofo adrenalin. No volunteers, however, developed malaria fever while mepacrine was being taken.

Immediately following these experimental re sults a military conference was held in Queenslandô and all the measures suggested were adopted. In consequence, malignant tertian malaria and응 blackwater fever practically disappeared in the South West Pacific and though benign tertian and․ㅡ․ quartan malaria frequently followed cessation off mepacrine administration, fatalities were almost unknown. Comparable results were obtained amongst British troops in Burma but the incidencew of malaria in Japanese troops persisted at a very

high level throughout the whole war.
Chloroquine diphosphate was discovered and patented in Germany in 1938 and was synthesized: in U.S.A. during the war. Fairley et al. ${ }^{6}$ found it ${ }^{0}$ prevented symptoms of malaria developing in 
volunteers experimentally infected with M.T. malaria while taking o.r g. daily. Subinoculations on the eighth day were always positive, but after ceasing the daily dose on the 23 rd day, parasites had been destroyed and they never reappeared in blood smears. On the 8ist day the volunteers were again successfully reinfected, no evidence of premunity being found. Similar results were recorded in a few volunteers taking $50 \mathrm{mg}$. of chloroquine daily. As with mepacrine, benign tertian infections were completely suppressed with chloroquine, but relapses generally occurred within two months of cessation of drug prophylaxis. Davey and Robertson ${ }^{4}$ in Nairobi working on Army volunteers confirmed Fairley's findings that chloroquine in a dosage of $50 \mathrm{mg}$. daily was an effective prophylactic against M.T. malaria, eliminating the infection entirely.

Proguanil was discovered by Curd, Davey and Rose $^{3}$ to be effective in bird malaria. It was investigated in man by Adams et al. ${ }^{1}$ and by Fairley et al. ${ }^{6}$ In M.T. malaria it was found to be an excellent prophylactic drug destroying the preerythrocytic forms in the liver before they reached the blood stream. Subinoculation on the eighth day was always negative in these patients, whereas with mepacrine and chloroquine it was invariably positive, the parasites being destroyed by these drugs in the blood stream later. With B.T. and quartan infected volunteers, fever failed to develop while they were taking proguanil, but generally appeared within two months when drug administration ceased. The dosage of proguanil for nonimmune adults is $100 \mathrm{mg}$. daily; for children under 6 years it is $25 \mathrm{mg}$. daily and from 6 to 12 years it is $5^{\circ} \mathrm{mg}$. daily.
Lapudrine. Robertson ${ }^{9}$ reported that $15 \mathrm{mg}$. once a week is an effective prophylactic.

Pyrimethamine (daraprim). Rollo ${ }^{10}$ described tests of antimalaria activity against the blood forms and exo-erythrocytic forms of $P$. gallinaceum and also against the blood forms of $P$. berghei. Goodwin $^{8}$ stated that good reports on the suppressive action of pyrimethamine had been received from the Belgian Congo, the Gambia, IndoChina and Tunisia but less so from Malaya. Foy and Kondi ${ }^{7}$ investigated the action of pyrimethamine on the gametocytes of $P$. falciparum. They showed it rendered gametocytes uninfective to $A$. gambiae. It is now recognized that the best value of paludrine and daraprim is for prophylactic and suppressive purposes since both these drugs act slowly in the febrile attack. Daraprim in a dosage of $25 \mathrm{mg}$. per week is given to adults; children under the age of 6 years receive $6.25 \mathrm{mg}$. per week and those from 6 to 12 years receive 12.5 mg. per week.

\section{REFERENCES}

I. ADAMS, A. R. D., MAEGRAITH, B. G., KING, I. D TOWNSHEND, R. H DAVEY, $R, H$, and HARVARD, R. E. (1945), Ann. trop. Med. Parasit., 39, 225.

2. CURD, F. H. S., DAVEY, D. G., HENDRY, J. A., and ROSE, F. L. (1950), Brit. F. Pharmacol., 5, 438.

3. CURD, F. H. S., DAVEY, D. G., and ROSE, F. L. (1945), Ann. trop. Med. Parasit., 39, 208.

4. DAVEY, D. G., and ROBERTSON, G. I. (1957), Trans. roy Soc. trop. Med. \& Hyg., 51, 463, 502.

s. FAIRLEY, N. H., et al. (1945), Ibid., 38, 31 I.

6. FAIRLEY, N. H., et al. (1946), Ibid., 40, 229.

7. FOY, H., and KONDI, A. (1952), Ibid., 46, 370.

8. GOODWIN, R. G. (1952), Ibid., 46, 485 .

9. ROBERTSON, G. I. (1957), Ibid., 5I, 457.

10. ROLLO, I. M. (1952), F. Amer. med. Ass., 46, 474.

11. REPORTS published by authority of Council of Pharmacy(1952), Ibid., 149, 1558. 